\title{
MEMORIAS Y NARRATIVAS DE LOS DOCENTES: CARTAS PEDAGÓGICAS SOBRE LA CULTURA AFRO-BRASILEÑA Y AFRICANA EN EL CURRÍCULO ESCOLAR
}

\author{
TEACHERS' MEMORIES AND NARRATIVES: PEDAGOGICAL LETTERS ABOUT \\ AFRO-BRAZILIAN AND AFRICAN CULTURE IN THE SCHOOL CURRICULUM
}

\author{
Ercília Maria Angeli Teixeira de Paula \\ Universidade Estadual de Maringá, Brasil \\ https://orcid.org/0000-0002-8619-7558 \\ erciliangeli@yahoo.com.br \\ Ingrit Yasmin Oliveira da Silva Batista \\ Universidade Estadual de Maringá, Brasil \\ https://orcid.org/0000-0003-4880-7199 \\ ingrityasmin_@hotmail.com \\ Mercy Lucero Cepeda Mesa \\ Universidade Estadual de Maringá, Brasil \\ https://orcid.org/0000-0003-0447-5858 \\ lucem03@gmail.com
}

Recibido: 02-12-2019. Revisado: 13-12-2019 Aceptado: 13-03-2020

\begin{abstract}
Resumen: El objetivo de este artículo es verificar, a través de las narraciones escritas en cartas pedagógicas de docentes y estudiantes, que representaciones existen en las prácticas pedagógicas y en los currículos sobre la cultura Afro-Brasileña y Africana en la escuela. La investigación se llevó a cabo con cuatro docentes y cuatro estudiantes de cuarto grado de los años iniciales de educación primaria de cuatro escuelas diferentes, ubicadas en la ciudad de Campo Mourão-Paraná (Brasil). La investigación es de carácter cualitativo y los instrumentos metodológicos utilizados fueron las cartas pedagógicas. Como resultado, se observa que las narrativas que circulan en la rutina escolar deben ser discutidas. Con esta investigación, a través del análisis de las letras, parece que el trabajo con la cultura Afro-Brasileña es abordado de manera fragmentada. Por lo tanto, la necesidad de fomentar estudios sobre la importancia de la representación de etnia negra, que los maestros, los alumnos y todo el equipo escolar deben hacer visible, a fin de que los niños puedan acceder a otras representaciones y culturas.
\end{abstract}

Palabras clave: Narrativas, cartas pedagógicas, cultura afro-brasileña y africana.

\begin{abstract}
The objective of this article was to verify, through the narratives written in pedagogical letters from teachers and students, which representations about Afro-Brazilian and African Culture are in the educational practices and in the school curricula. The research was carried out with four teachers and four students from the 4th Year of the Initial Years of Elementary Education from four different schools, located in the city of Campo Mourão-PR. This qualitative research used methodological instruments as teaching letters. As a result, it is observed that the narratives that circulates in the school routine need to be discussed. Findings of this research, through the analysis of the letters, appears the work with Afro-Brazilian Culture is approached in a fragmented way. Therefore, there is a need to foster studies on the importance of black representation, which should be made visible to teachers, students and the entire school team, in order to allow children access other representations and cultures.
\end{abstract}

Keywords: Narratives, pedagogical letters, afro-brazilian and african culture.

e-ISSN:2172-7910

Doi: http://doi.org/10.25267/Hachetetepe.2020.i20.5

Universidad de Cádiz 
Cómo citar este artículo: Paula, E.M.A T., Batista, I.Y.O S y Mesa, M.L.C.(2020). Memorias y narrativas de los docentes: cartas pedagógicas sobre la cultura afro-brasileña y africana en el currículo escolar. Hachetetepé. Revista científica en Educación y Comunicación, (20), 31-43.doi. http://doi.org/10.25267/Hachetetepe.2020.i20.5

\section{INTRODUCCIÓN}

La escritura hecha a mano ha perdido espacio por el uso de los computadores/ordenadores, tablets y teléfonos celulares/móviles, debido a la facilidad de las personas hoy en día para escribir y poder observar las correcciones gramaticales y ortográficas del idioma. La escritura es una forma utilizada en el entorno educativo, durante las evaluaciones, los exámenes de ingreso, entre otros momentos. Sin embargo, esta forma de escritura está desapareciendo en la sociedad contemporánea.

Las cartas informales son narrativas que revelan subjetividades, percepciones y valores sobre el mundo y la sociedad. Se debe estimular la redacción de cartas, tanto en las escuelas como en la producción de investigación. A través de las cartas, las personas narran sus vidas, recuerdos e historias.

Este artículo tiene como objetivo relatar una investigación desarrollada en la disciplina trabajo escolar y producción social del Conocimiento, del Programa de Postgrado en Educación de la Universidad Estatal de Maringá - UEM, ofrecida para el Máster en el segundo semestre de 2018. La investigación se llevó a cabo con profesores y alumnos de primaria en una ciudad del interior de Paraná (Brasil). Se solicitó la producción de cartas sobre temas relacionados con las formas de trabajo en las escuelas y el plan de estudios sobre cuestiones étnico-raciales.

El estudio se llevó a cabo con cuatro docentes y cuatro estudiantes del cuarto año de la escuela primaria, de cuatro escuelas públicas diferentes, en el municipio de Campo Mourão - PR. El objetivo era verificar, a través de las narraciones escritas en cartas de profesores y estudiantes, qué representaciones en prácticas pedagógicas y planes de estudio sobre la cultura Afro-Brasileña y Africana se evidencian en la escuela.

El tema de las cartas se enfocó a determinar el trabajo con la cultura Afro-Brasileña y Africana en el currículo escolar y como las docentes desarrollaron sus prácticas a favor de la implementación de la Ley 10.639 / 03 (Brasil, 2003), que se justifica por la necesidad de abolir las desigualdades sociales e históricas que afectan a la población negra. Con respecto a los estudiantes, la propuesta era verificar si esta ley se está cumpliendo en las escuelas.

La autorización para las profesoras se realizó, a través de un formulario estándar proporcionado por las investigadoras a todas las participantes. Los mayores de edad firmaron y los responsables de menores firmaron por ellos. El desarrollo de la investigación se produjo después de que los participantes firmaron el formulario de consentimiento libre e informado por las participantes de la pesquisa.

Las reflexiones se llevaron a cabo con profesores y estudiantes a través de la siguiente pregunta: ¿Cómo se representa a las personas negras en el plan de estudios de las escuelas donde estudio y en la sociedad actual? La investigación y los procedimientos e instrumentos metodológicos utilizados para la producción de las cartas fueron de carácter cualitativo. Los fundamentos teóricos se centraron en los Estudios Culturales (Silva, 2011), porque considera que el plan de estudios de la escuela debe resaltar la diversidad, en vista de los estudios de género, sexualidad y etnia, para que no dar paso a

e-ISSN:2172-7910

Doi: http://doi.org/10.25267/Hachetetepe.2020.i20.5

Universidad de Cádiz 
posturas silenciadoras en el entorno escolar de los estudiantes, grupos considerados minorías.

El artículo se sistematizó de la siguiente manera: introducción, presentada aquí; metodología de investigación utilizando cartas pedagógicas; presentación de las narraciones de los maestros sobre cómo se representa a la población negra en el currículo escolar; las imágenes que los alumnos tienen sobre los maestros de los primeros años de la escuela primaria, el análisis de las cartas pedagógicas y, por último, las consideraciones finales de este estudio. En este sentido, se pretende colaborar con los debates y reflexiones sobre la formación de docentes y estudiantes basados en el uso de las cartas como estrategia de investigación.

\section{CARTAS COMO INSTRUMENTOS DE PESQUISA}

Actualmente, en el siglo XXI, no es muy común usar cartas como estrategia de investigación en la vida escolar cotidiana. Esto se debe al uso exacerbado de la tecnología que ocurre rutinariamente en las escuelas y la sociedad. Los recursos tecnológicos, aunque importantes, su uso como estrategia en el aula, revela la ausencia de estímulo para la escritura. Según Netto, Spagnolo, Florentino, Amaral, Zancas y Portal (2012, p.16) "Escribir es una forma de expresión a través de símbolos. A través del acto de escribir es posible desarrollar diferentes competencias y habilidades, además de tener una gran representatividad como medio de comunicación, inevitablemente en la era digital y virtual en la que vivimos actualmente".

La producción de cartas implica tiempo para escribir el texto, reflexionar sobre lo que está escrito y analizar el producto final para enviar estas cartas. En los tiempos modernos, debido a la velocidad con la que avanza la sociedad, es muy poco el tiempo que se le dedica a la escritura.

Los autores Sousa y Cabral (2015) resaltan que la carta tiene un lenguaje determinado según la intención comunicativa entre pares y también es una herramienta educativa de fácil acceso. Con respecto al uso de la escritura de cartas, Paula (2018, p.17) destaca que:

Escribir cartas era una actividad propuesta por los educadores en las escuelas y no se limitó a la capacitación de un tipo de género textual destinado a escribir el examen de ingreso. La escritura de las cartas fue más allá de las aulas, principalmente las cartas de amor, escritas cuidadosamente por parejas enamoradas. Para los analfabetos, había escribas, responsables de escribirlos y compartir las emociones de las personas que no tenían acceso a los procesos escolares. Las cartas formales, informales y amorosas formaban parte de la vida cotidiana de las personas. Esperar cartas y recibirlas también fueron momentos expresivos. El mundo era conocido a través de las descripciones de amigos y familiares. La espera, el tiempo, la calidad del texto, la caligrafía de las letras, los sobres, la tinta de los bolígrafos, todos estos elementos fueron cuidadosamente preparados por quienes escribieron estas cartas para impresionar al lector. La escritura fue auténtica, creativa, ficticia o realista. Estos aspectos dependían del contexto y la situación. Las cartas eran documentos y testimonios de un tiempo lejano que parece no volver nunca.

Cabe resaltar que, según el autor, existía una planificación al escribir las cartas, desde el papel en el que se escribían hasta el color del esfero. Los que escribieron y los

e-ISSN:2172-7910

Doi: http://doi.org/10.25267/Hachetetepe.2020.i20.5

Universidad de Cádiz 
que recibieron se conectaron incluso desde la distancia por esos escritos. Sin embargo, es una pena que estén desapareciendo de la vida cotidiana de las personas.

Con respecto a la escuela, Trilla, Ghanem, Arantes, (2008, p.35) enfatizan que “"...] el sistema debe ser abierto, flexible, evolutivo, rico en cantidad y diversidad de ofertas y medios educativos y un sistema educativo solo puede ser así si realmente incorpora el sector no formal y considera y valora lo informal".

Es evidente la necesidad de considerar otras formas de aprendizaje, que proporcionarán un mejor desarrollo y rendimiento de los estudiantes. En este sentido la escuela debe ser un entorno de aprendizaje abierto al diálogo, que tenga sentido en y para la vida de los sujetos. Un espacio que, en lugar de excluir a la familia, mantenga una relación de reciprocidad y brinde a los estudiantes la posibilidad de convertirse en ciudadanos críticos, participativos y autónomos tanto en la escuela como en la sociedad.

En sus escritos, Gadotti (2011) promueve que la carta permita la complicidad entre quienes escriben y los que leen, además, menciona que quien escribe cartas invita al diálogo, siendo una poderosa herramienta pedagógica para la enseñanza.

La narrativa es parte de la historia humana y, por lo tanto, debe estudiarse dentro de sus contextos sociales, económicos, políticos, históricos y educativos. Es común escuchar a través de diferentes narrativas que los seres humanos son, por naturaleza, narradores de historia y que generaciones y generaciones repiten este acto casi involuntariamente entre sí unos a otros (Sousa y Cabral, 2015, p.150).

Además, en esta investigación, las cartas se usaron como narrativas porque los participantes contaron sobre sus historias personales y profesionales y la relación que tienen con la cultura Afro-Brasileña y Africana.

Para la recopilación de datos, se informó a los participantes sobre los procedimientos éticos de la investigación. Se aclararon los objetivos del trabajo y se dieron los consentimientos informados a los maestros y estudiantes participantes, para tener presente la veracidad y ética de la investigación y para autorizarla. Los docentes participantes firmaron el consentimiento informado para participar en este trabajo y los tutores legales de los menores firmaron por ellos.

Los criterios utilizados para la selección de los participantes fueron: una profesor(a) y un alumno(a) de cuatro escuelas diferentes ubicadas en la región periférica del municipio de Campo Mourão - PR. Debido a la falta de docentes hombres y negros en las escuelas elegidas, el trabajo fue realizado solo por maestras, por lo que también se eligieron solo alumnas.

La intención era verificar si las prácticas pedagógicas de los docentes tienen similitudes o diferencias y cuál es la forma en que se muestra a los estudiantes negros, porque son escuelas ubicadas cerca unas de otras. El profesor y/o el alumno deben ser negros. A partir de eso, se pidió a los maestros que describieran su práctica pedagógica vinculada a la cultura Afro - Brasilera y Africana.

Con respecto a los estudiantes, se les pidió que escribieran qué imágenes tenían sobre los profesores y narraran la forma en que fueron tomados en cuenta en el curriculo escolar.

Con la implementación de la Ley 10.639/03, la enseñanza de la historia y cultura Afro - Brasilera y Africana en el currículo escolar se hizo obligatoria (Brasil, 2003). Por lo tanto, existe la necesidad de acciones didácticas en las diferentes etapas de las

e-ISSN:2172-7910

Doi: http://doi.org/10.25267/Hachetetepe.2020.i20.5

Universidad de Cádiz 
modalidades de aprendizaje y enseñanza, que deben llevarse a cabo durante todo el año escolar y no de manera fragmentada, a fin de valorar la historia y la cultura de las personas en sus diversas diferencias. Becker (2012, p. 24) fomenta que:

El profesor desarrollará su enseñanza cada día, racionalizando su proceso de aprendizaje. Los estudiantes construirán su "discurso" cada día, enseñando a sus colegas y al maestro nuevos conocimientos, nociones, conceptos, objetos culturales, teorías, comportamientos. A menudo harán preguntas banales, pero otras que desafiarán al profesor.

Por lo tanto, el profesor cree que el alumno siempre podrá aprender y enseñar algo nuevo. El proceso de valoración del maestro permite a los estudiantes crear nuevos conocimientos, nuevas respuestas a viejas preguntas, a fin de construir un mundo de respeto por los demás, teniendo en cuenta todos los tipos de conocimiento válidos, aprendidos en la educación formal o en otros contextos.

A través del análisis de las narrativas de las cartas, fue posible verificar un diálogo entre los participantes de la investigación sobre cómo entienden el papel del profesor y las instituciones en la inserción del plan de estudios sobre la cultura Afro - Brasileña y Africana.

Optamos por el criterio de elegir nombres ficticios, con las letras secuenciales del alfabeto para preservar la identidad de los maestros y estudiantes que participan en la investigación. Por lo tanto, los nombres de los cuatro profesores y los cuatro estudiantes están de acuerdo con las primeras cuatro letras del alfabeto.

A continuación, presentamos dos tablas que se refieren a la composición general con información de docentes y estudiantes.

\section{Tabla 1}

Datos informativos sobre los docentes participantes

\section{DATOS INFORMATIVOS DE LOS DOCENTES}

\begin{tabular}{|c|c|c|c|c|c|}
\hline Nombre & Edad & $\begin{array}{l}\text { Formación } \\
\text { Académica }\end{array}$ & $\begin{array}{l}\text { Institución } \\
\text { de formación }\end{array}$ & $\begin{array}{c}\text { Tiempo } \\
\text { de } \\
\text { actuación } \\
\text { en la } \\
\text { educación }\end{array}$ & $\begin{array}{c}\text { Posgrado Lato } \\
\text { Sensu o Stricto } \\
\text { Sensu }\end{array}$ \\
\hline Aline & 29 & $\begin{array}{l}\text { Pedagogía/ } \\
\text { Presencial }\end{array}$ & Pública & 3 años & $\begin{array}{c}\text { Lato Sensu en } \\
\text { Educación Especial }\end{array}$ \\
\hline Bianca & 48 & $\begin{array}{l}\text { Pedagogía/ } \\
\text { Presencial }\end{array}$ & Pública & 6 años & $\begin{array}{c}\text { Lato Sensu en } \\
\text { Psicopedagogia/ } \\
\text { Neuropedagogia }\end{array}$ \\
\hline Caroline & 26 & $\begin{array}{l}\text { Pedagogía/ } \\
\text { Presencial }\end{array}$ & Particular & 3 años & $\begin{array}{c}\text { Lato Sensu en } \\
\text { Psicomotricidad/ } \\
\text { Educación Especial }\end{array}$ \\
\hline Daniela & 45 & $\begin{array}{l}\text { Magistério/ } \\
\text { Pedagogía/ } \\
\text { Presencial }\end{array}$ & Pública & 10 años & $\begin{array}{c}\text { Lato Sensu en } \\
\text { Educación Especial }\end{array}$ \\
\hline
\end{tabular}

Fuente: Tabla sistematizada por los autores

e-ISSN:2172-7910

Doi: http://doi.org/10.25267/Hachetetepe.2020.i20.5

Universidad de Cádiz 
Tabla 2

Datos informativos sobre los estudiantes participantes

DATOS INFORMATIVOS DE LOS ESTUDIANTES

\begin{tabular}{cccc}
\hline Nombre & Edad & $\begin{array}{c}\text { Formación } \\
\text { Académica }\end{array}$ & $\begin{array}{c}\text { Institución de } \\
\text { formación }\end{array}$ \\
\hline Alice & 10 & $\begin{array}{c}\text { Estudiante de } 4^{\circ} \\
\text { Año }\end{array}$ & Pública \\
\hline Bruna & 10 & $\begin{array}{c}\text { Estudiante de } 4^{\circ} \\
\text { Año }\end{array}$ & Pública \\
\hline Camila & 9 & $\begin{array}{c}\text { Estudiante de } 4^{\circ} \\
\text { Año }\end{array}$ & Pública \\
\hline Daiane & 9 & Estudiante de $4^{\circ}$ & Pública \\
\hline
\end{tabular}

Fuente: Tabla sistematizada por los autores

Todas las docentes entrevistadas son concursadas por el municipio de Campo Mourão - PR, y trabajan en grupos de cuarto año de la escuela primaria. Tienen entre 26 y 48 años, tienen un título en pedagogía y un título de posgrado en educación en Lato Sensu.

Los estudiantes participantes estudian en el $4^{\circ}$ año de la escuela primaria. Tienen entre 9 y 10 años y estudian en escuelas públicas municipales.

Luego, se analizaron las representaciones de los maestros sobre cómo se representa a los negros en el plan de estudios de las escuelas y cuál es la imagen de los estudiantes sobre los maestros.

\subsection{Narrativas: ¿cómo se representan los negros (a) en los planes de estudio escolares?}

Para que los niños se acerquen y aprendan sobre otras culturas, es necesario que el trabajo sobre temas étnico-raciales se vincule a las actividades diarias durante el año escolar con temas dirigidos a la memoria de las personas negras. Sin embargo, los negros sólo recuerdan fechas específicas, como el "Día de la Abolición de la Esclavitud" o el "Día Nacional de la Conciencia Negra", celebrado el 20 de noviembre y el 13 de mayo. respectivamente.

Sobre cómo se representan los negros en los planes de estudio escolares, la maestra Aline destacó:

Soy negro (a), por lo que vale la pena mencionar aquí que creo y posiciono que debemos valorar al ser humano, y de esta manera valorar la historia y cultura Afro - Brasileña y Africana durante el año escolar, trato de desemvolver y trabajar de acuerdo con La planificación anual de la secretaría de educación. [...] Creo y trabajo de una manera que mis alumnos respetan y valoran a las personas como seres humanos, y no de acuerdo con su color de piel, raza, sexo, entre otros (ALINE, 2018, extracto de sic extraído de la carta)

e-ISSN:2172-7910

Doi: http://doi.org/10.25267/Hachetetepe.2020.i20.5

Universidad de Cádiz 
Gomes (2003, p.171) reverbera la necesidad de acciones didácticas centradas en este tema y destaca:

Construir una identidad negra positiva en una sociedad que, históricamente, ha enseñado a los negros, desde una edad muy temprana, que para ser aceptado es necesario negarse a sí mismo, es un desafío que enfrentan los Brasileros negros. ¿Somos conscientes de este problema en la escuela? ¿Incorporamos esta realidad de manera seria y responsable cuando discutimos, en los procesos de formación docente, sobre la importancia de la diversidad cultural?

El párrafo anterior nos hace pensar si las escuelas realizan un trabajo efectivo para la enseñanza de la cultura Afro-Brasileña y Africana. La docente Bianca menciona:

En la escuela donde trabajo, escuché varios informes de preconceptos contra ellos (personas negras), incluso de un padre cuestionando si ella era maestra. [...] el año pasado [...] participé en cursos de capacitación continua que abordaron el tema y fue extremadamente importante para mi capacitación» (Bianca, 2018, sic párrafo extraído de la carta)

A través de la carta de la docente, queda claro que la persona negra todavía se ve como inferior a la persona blanca, cuando el padre de un estudiante pregunta si ella es docente, debido a su color, es un maestro. Otro aspecto importante se refiere a los cursos de educación continua en los que la maestra menciona que participó y que marcó una diferencia en su ejercicio docente. Por lo tanto, Esteve (2004, p.160), destaca que:

La formación inicial y continua de nuestros profesionales es el elemento esencial para mejorar la calidad de nuestros sistemas educativos. La formación inicial debe comenzar a preparar a los futuros docentes para los desafíos de la nueva sociedad del conocimiento, y para enfrentar, en las aulas, los nuevos problemas que surgen de los cambios profundos... Por lo tanto, el primer desafío a enfrentar es la capacitación. de nuestros docentes

Para las personas negras es mas difícil ocupar posiciones privilegiadas en la sociedad debido a la falta de oportunidades y los prejuicios que aún existen. Con respecto a la práctica pedagógica sobre las personas negras en la escuela, la maestra Caroline dice:

Se hace superficialmente. Creo que no hay silencio, pero es algo poco trabajado y desarrollado en el entorno escolar. Durante el año académico, se trata el tema del respeto en general en relación con los preconceptos y la diversidad, en el que los estudiantes exponen sus ideas en forma de debates, pero no solo o específicamente sobre la cultura Afro-Brasileña y Africana. Se trata globalmente y especialmente en días festivos. Además de las conversaciones, estamos trabajando con videos, documentales, películas y actividades en grupos para que haya una mejor socialización, porque en la clase hay una diversidad de problemas familiares y eso se refleja en la escuela, pero es un trabajo largo y debe continuar. Hice un trabajo de lectura y debate con libros sobre sentimientos [...] que a los estudiantes les gustaban mucho y expuse sus ansiedades y conflictos, incluida la sensación de no ser aceptados por sus colegas debido al color (Caroline, 2018, sic Extraido de la carta )

e-ISSN:2172-7910

Doi: http://doi.org/10.25267/Hachetetepe.2020.i20.5

Universidad de Cádiz 
A través de la carta, la docente enfatiza que las personas negras son visivilizadas en el plan de estudios de manera artificial. También señala que las escuelas trabajan en general en temas relacionados con este asunto. Sin embargo, solo durante las fechas conmemorativas se resalta el tema. Ella menciona los problemas familiares de los estudiantes, que interfieren con el aprendizaje escolar.

Es esencial que haya una buena relación entre la familia y la escuela que tenga como objetivo un mejor trabajo educativo, ya que ambos tienen como objetivo principal el desarrollo y el aprendizaje del estudiante. Según Cunha (2000, p.447):

La familia y la escuela son instituciones sociales que giran en torno al mismo centro, el estudiante, ya sea un niño o joven o, en algunos casos, adultos: el ser educativo, el individuo que se desarrolla desde la infancia hasta la madurez, el ser social involucrado en las temas culturales y políticas de su entorno.

El entorno familiar, así como su relación con el aprendizaje escolar, es fundamental tanto para el proceso de aprendizaje como para los problemas de formación cultural y social del alumnado.

Cunha (2000) promueve que la familia y la escuela señalen aspectos mutuos que son insuficientes. Por lo tanto, ambos están en conflicto, lo que demuestra que las familias no están satisfechas con las escuelas y viceversa. La docente Daniela describe sobre las personas negras en el plan de estudios y en la rutina escolar de la siguiente manera:

En la escuela donde trabajo, se han llevado a cabo actividades relacionadas con la apreciación de la cultura Afro-Brasileña y Africana, desde principios de año. Especialmente en las clases de Filosofía, haciendo espacio para el círculo de conversación, dinámica, lecturas, narración de cuentos y música, además de la fecha conmemorativa. Al abordar el tema, algunos estudiantes dijeron que ya habían sufrido preconceptos y que habían visto personas en la calle con prejuicios contra otros. Y la misma mujer estaba asustada y silenciada. Nunca cambiaría la forma de creer que todavía hay esperanza y que podemos marcar la diferencia en la vida de un niño (Daniela, 2018, sic Extraido de la carta)

A través de los escritos de la docente, está claro que para las personas negras todavía es difícil hablar sobre lo que les preocupa y los perturba. Para Sousa y Cabral (2015, p.150):

Es este carácter flexible de la memoria, entretejido en la relación con el otro, lo que permite a los sujetos rehacer sus historias, en sus recuerdos, resistiendo lo que les molesta, agregando hechos de su deseo de que hubiera sido diferente, como nuevas posibilidades para sus experiencias. Por lo tanto, entendemos que las situaciones narradas son revividas y reelaboradas en el proceso de recuperación.

Es común que los hombres y mujeres negros silencien sus emociones por miedo. Como docentes, es necesario creer y luchar por una sociedad que respete todas las diferencias existentes a través de prácticas pedagógicas que aborden la visibilización de la diversidad nacional. 


\subsection{La imagen que los estudiantes tienen sobre los docentes en los primeros años de la escuela primaria}

La educación primaria, durante muchos años estuvo dirigida por personas con mayor poder adquisitivo y niños excluidos de bajos ingresos, y niños negros, fue reemplazada y extendida a toda la población.

Reanudando la dinámica histórica, en los últimos 80 años, las oportunidades de acceso y permanencia en el sistema escolar se han expandido significativamente para amplios sectores de la población (Beisegel, 1986), convirtiendo la educación básica obligatoria a fines del siglo XX. prácticamente universal con respecto al acceso (Oliveira, 2007, p.666)

Dado lo anterior, está claro que hubo muchos avances educativos que ocurrieron en Brasil. Sin embargo, aún queda mucho por hacer para prevenir las desigualdades sociales en el país, como lo menciona Oliveira (2007, p.682)

[...] la desigualdad y la exclusión permanecen. No es por esta razón que la educación primaria ha dejado de ser una etapa que produce desigualdad educativa. Además, la discriminación de ayer sigue siendo la discriminación de hoy. Pero la desigualdad que existe hoy ya no es la misma, ni ocurre en los mismos términos que en el pasado.

La universalización de la educación primaria fue un avance importante en el área de la educación. Sin embargo, todavía existe la exclusión de aquellos considerados fuera de los estándares socialmente establecidos. En el ambiente escolar, los maestros deben actualizarse, buscar nuevos conocimientos para enfrentar los desafíos de hoy. La educación autoritaria, basada en la enseñanza sin diálogo, debe superar los paradigmas establecidos por la sociedad.

En el ambiente escolar, los maestros deben actualizarse, buscar nuevos conocimientos para enfrentar los desafíos de hoy. La educación autoritaria, basada en la enseñanza sin diálogo, debe superarse.

El investigador Arroyo (2000, p.10), al escribir sobre la imagen que los estudiantes tienen sobre los maestros, reflexiona sobre el poco conocimiento que los educadores tienen sobre su propia historia. Y además resalta:

La educación nos recuerda a la escuela, no a sus profesionales, educadores. Estos no pueden ser la referencia, sino la institución escolar. Incluso la historia de la educación estudiada por los docentes es la historia de la escuela, de los sistemas educativos y no de su historia profesional, de su conocimiento profesional.

El autor brinda la posibilidad de que los maestros piensen en su educación. La estudiante Alice, informó sobre la imagen que tiene sobre los maestros.

En mi escuela, creo que la apreciación de la cultura Afro-Brasileña y Africana ha sido muy buena, porque nunca se observo un preconcepto, sé que hay muchos prejuicios y racismo, nunca supe que alguien sufrió y guardó silencio al respecto. Hace poco, vimos la esclavitud y su abolición, con la Ley del Libre Vente, la Ley de los Sexagenarios y la Ley Dorada, la imagen que tengo de los maestros es una imagen muy clara, tengo una gran admiración, porque nos enseñan todo lo que necesitaremos para el futuro, sin profesores no se forma ninguna profesión, luchan incansablemente para enseñar mejor y

e-ISSN:2172-7910

Doi: http://doi.org/10.25267/Hachetetepe.2020.i20.5

Universidad de Cádiz 
a menudo no son reconocidos, golpeados y humillados por las autoridades (Alice, 2018, sic párrafo extraído de la carta).

La alumna demuestra admiración a los profesores y revela la precariedad de sus condiciones de trabajo. Además, aborda la necesidad de una mirada cuidadosa a esta clase que no se valora como debería. La estudiante Bruna señaló que en la escuela donde estudia tiene:

[...] estudiantes y empleados negros no hay forma de silenciarlos, ya que tienen el mismo derecho a expresarse que los demás, sin embargo, en algunas situaciones ya he presenciado a colegas negros que sufren bullying [...] Los profesores son personas que dedicaron y dedican su tiempo y estudio a enseñarnos (Bruna, 2018, sic párrafo extraído de la carta)

Por lo tanto, la necesidad de abordar cuestiones relevantes está justificada. Los prejuicios sociales siguen estando inmersos en la sociedad, por lo que es necesario defender como educadores que la población negra y blanca tienen los mismos derechos que los ciudadanos y no pueden silenciar sus sentimientos. Además, cuando los docentes dedican su tiempo a enseñar, aportan numerosos beneficios a sus alumnos.

[...] la profesora nos enseña la historia de los esclavos de Brasil y toda su cultura, que hasta hoy son valorados como gastronomía, bailes, artesanías y algunos deportes y costumbres, etc. [...] Nunca he sido testigo de ningún acto de prejuicio o silenciar a las personas negras en mi escuela [...] como una buena maestra, entiendo todo lo que explica $\mathrm{y}$ un trabajo consolidado que forma todas las profesiones, pero no se valora como debería ser (Camila, 2018, sic párrafo extraído de la carta)

En vista de esta afirmación sobre las relaciones educador-alumno, Freire (1997, p.19) presenta la siguiente afirmación de que:

El aprendizaje del profesor, cuando enseña, tiene lugar cuando el maestro, humilde, abierto, está permanentemente disponible para repensar el pensamiento, para revisarse en sus posiciones; en el que trata de involucrarse con la curiosidad de los estudiantes y los diferentes caminos y veredas por los que los atraviesa.

En esta perspectiva, los educadores deben hacer que los estudiantes reflexionen sobre sus acciones, motivarlos hacia la curiosidad y la búsqueda constante del conocimiento, encontrando en la investigación una posibilidad de construir el saber. La estudiante Daiane reveló:

En mi escuela hablamos sobre silenciamiento, amenazas, racismo y crimen. La imagen que tengo de los negros es la misma que la de todos los demás, excepto que todos tienen su propio camino. (...) cuando comencé en la escuela en Curitiba tenía un color más oscuro en mi clase, así que comencé a sufrir racismo, pero no me quedé callado, le dije a mi maestra. Después de que la niña dejó mi escuela, dejé de sufrir racismo (Daiane, 2018, extracto de la carta) 
El texto mencionado hace eco de la necesidad de acciones didácticas que aborden cuestiones raciales en diferentes etapas de las modalidades de aprendizaje y enseñanza. Silva (2015, p.57) menciona que:

Para algunos, los prejuicios raciales pueden considerarse un tema desactualizado. ¿Todavía tenemos que hablar? ¡Sí, necesitamos hablar sobre prejuicios raciales! Ignorar los episodios del pasado y no debatir abiertamente el tema es descuidar los esfuerzos de todos aquellos que han luchado por el cambio. No hablar de la situación de las mujeres negras es ignorar su papel como constructoras de la historia, es ignorar su lucha por sobrevivir dentro de una sociedad donde el prejuicio racial se consideraba algo común.

Sobre la base de esta suposición y este trabajo de investigación llevado a cabo a través de cartas pedagógicas, fue posible mostrar cómo las mujeres negras continúan la búsqueda de la igualdad racial al narrar sus historias personales y profesionales, lo que destaca el estímulo para luchar por sus derechos civiles como mujeres, especialmente negras.

\section{RESULTADOS DEL ANÁLISIS DE LAS CARTAS PEDAGÓGICAS}

Para cumplir con los objetivos de la investigación, se analizaron cuatro cartas de maestros y cuatro cartas de estudiantes, una elegida por el maestro y otra por cuatro escuelas diferentes para esta investigación.

Las escuelas seleccionadas provenían de instituciones públicas que ofrecen educación infantil y primaria, todas ellas ubicadas en Jardim Lar Paraná, bajo la dirección de maestros del mismo personal de empleados municipales que fueron elegidos en la elección libre y directa para el puesto de directores en el segunda semestre del año 2017 para actuar frente a la gerencia entre los años 2018-2021. La mayoría de los estudiantes en estas escuelas son de familias de clase media baja y tienen un color marrón, seguido de blancos y negros, lo que lleva a repensar el color de la población que vive en el sur del país, principalmente blanco y marrón.

Por medio del análisis de los datos recopilados en las cartas fue evidente, que las escuelas realizan el trabajo de manera artificial. En cuanto a los docentes ellos escribieron en las cartas que buscan mejorar su ejercicio pedagógico y trabajar de acuerdo con la planificación anual de la secretaría de educación y abordar el tema siempre que sea posible a través de contenidos que fomenten la valorización de los seres humanos.

Además, resaltar que al trabajar con el tema de la cultura Afro-Brasileña en la escuela, los estudiantes relataron experiencias negativas de sus vidas, en las que sufrieron prejuicios y trataron de superar situaciones a través del diálogo con los estudiantes. vinculado a actividades grupales, películas y cuentos.

Los estudiantes mencionaron que han sido estigmatizados por la sociedad y que la imagen que tienen sobre las personas negras debe cambiar para que sean respetados. También informaron que ven a los maestros como aquellos que se dedican a la enseñanza, pero que no son valorados en la sociedad. Por lo tanto, tienen una imagen de admiración, lo que demuestra que durante el período escolar, fueron tratados con respeto en el entorno escolar. 


\section{CONSIDERACIONES FINALES}

Con el uso de las cartas de enseñanza como recurso, parece que docentes y estudiantes denunciaron momentos de difíciles de su vida por el hecho de ser raza Afro.

En sus escritos, también se enfatiza que se logró el objetivo de la investigación, teniendo en cuenta que las narrativas de los profesores sobre las prácticas pedagógicas sobre la cultura Afro-Brasileña y Africana en la escuela se han abordado para promover reflexiones, preguntas y discusiones con los estudiantes, y así superar las desigualdades que generan prejuicios. Con el análisis de las cartas, se observa que los maestros mostraron en sus escritos una preocupación por el tema del prejuicio racial que sufren los niños y niñas negros en las escuelas brasileras.

Con respecto a los estudiantes, es notable que en las escuelas donde estudiaron, no existió un plan de estudios centrado en las relaciones étnico-raciales, sin embargo, en las escuelas en las que actualmente estudian, la población negra se ve con respeto y aprecio. Además, la preocupación de los estudiantes por lo poco que es valorada la profesión.

Esta investigación promovió momentos que permitieron a los participantes percibir diferentes narrativas sobre la población negra y repensar sus historias de vida personales y profesionales.

Finalmente, se espera que el uso de las cartas como recurso metodológico se pueda introducir en los diferentes contextos, para proporcionar a los estudiantes posibilidades de acceso a representaciones culturales que no sean sólo desde una perspectiva eurocéntrica y por lo tanto, tenga en cuenta la importancia histórica de la cultura negra para la diversidad brasileña.

\section{REFERENCIAS BIBLIOGRÁFICAS}

Arroyo, M. (2000). Os saberes dos docentes disputam lugar nos currículos. Petrópolis: Vozes.

Becker, F. (2012). Educação e construção do conhecimento. Porto Alegre: Penso.

Brasil. Lei $n^{\circ} 10.639$, de 9 de janeiro de 2003. (2003). Altera a Lei $n^{\circ} 9.394$, de 20 de dezembro de 1996, que estabelece as diretrizes e bases da educação nacional, para incluir no currículo oficial da rede de ensino a obrigatoriedade da temática "História e Cultura Afro-Brasileira", e dá outras providências. Diário Oficial da União, Brasília, DF, 10 Enero.

Cunha, M. V. (2000). 500 Anos de Educação no Brasil. Belo Horizonte: Autêntica.

Esteve, J. M. (2004). A Terceira Revolução Educacional: a educação na sociedade do conhecimento. São Paulo: Moderna.

Freire, P. (1997). Professora sim, tia não: cartas a quem ousa ensinar. São Paulo: Editora Olho D'água.

Gadotti, M. (2011). Diálogo e intimidade. En E. Coelho., Pedagogia da Correspondência: Paulo Freire e a educação por cartas e libros, (pp.1314).Brasília: Liber Livros.

Gomes, N. L. (2003). Identidade Negra e Formação de Professores: um olhar sobre o corpo negro e o cabelo crespo. Educação e Pesquisa, 29(1), 167-182.

Netto, C., Spagnolo, C., Florentino, J., Amaral, L., Zancas, S. y Portal, L. (2012). Cartas: um instrumento desvelador que faz a diferença no processo educacional. Revista Educação por Escrito,3, 14-25

e-ISSN:2172-7910

Doi: http://doi.org/10.25267/Hachetetepe.2020.i20.5

Universidad de Cádiz 
Oliveira, R. P. (2007). Da Universalização do Ensino Fundamental ao Desafio da Qualidade: Uma Análise Histórica. Disponible en: http://www.scielo.br/pdf/es/v28n100/a0328100.pdf

Paula, E. (2018). Cartas pedagógicas: estratégias didáticas revisitadas para novos tempos. Curitiba: CRV.

Silva, F. D. (2015). Histórias Cruzadas" e a movimentação social feminina negra pelos direitos civis nos EUA nas décadas de 1950 e 1960. Centro Universitário Univates, Lajeado: RS. Disponible en : https://www.univates.br/bdu/bitstream/10737/839/1/2015FernandaDornelesdaSil va.pdf

Silva, T. T. (2001). Documentos de Identidade: uma introdução às teorias do currículo. Belo Horizonte: Autêntica.

Sousa, M. G. S. y Cabral, C. L. O. (2015). A narrativa como opção metodológica de pesquisa e formação de professores. Horizontes 33(2), 149-158.

Trilla, J., Ghanem, E. y Arantes, V. A (2008). Educação formal e não formal: pontos e contrapontos. São Paulo: Summus. 